\title{
Brightening of dark excitons in a single quantum dot containing a single magnetic ion
}

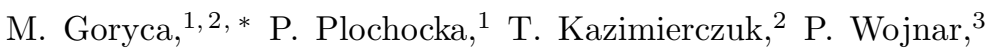 \\ G. Karczewski, ${ }^{3}$ J. A. Gaj, ${ }^{2}$ M. Potemski, ${ }^{1}$ and P. Kossacki ${ }^{1,2}$ \\ ${ }^{1}$ Laboratoire National des Champs Magnétiques Intenses, CNRS-UJF-UPS-INSA, 38042 Grenoble, France \\ ${ }^{2}$ Institute of Experimental Physics, University of Warsaw, ul. Hoża 69, 00-681 Warszawa, Poland \\ ${ }^{3}$ Institute of Physics, Polish Academy of Sciences, al. Lotników 32/46, 02-688 Warszawa, Poland
}

(Dated: November 12, 2018)

\begin{abstract}
A promising method to investigate dark exciton transitions in quantum dots is presented. The optical recombination of the dark exciton is allowed when the exciton state is coupled with an individual magnetic impurity (manganese ion). It is shown that the efficient radiative recombination is possible when the exchange interaction with the magnetic ion is accompanied by a mixing of the heavy-light hole states related to an in-plane anisotropy of the quantum dot. It is also shown that the dark exciton recombination is an efficient channel of manganese spin orientation.

PACS numbers: 73.21.La; 75.75.-c; 78.55.Et; 78.67.Hc
\end{abstract}

Semiconductor quantum dots (QDs) are among the most promising single-photon emitters [1-4]. They have potential applications in quantum information processing, and quantum telecommunications due to their seamless integration in semiconductor circuits, their robustness, and their relatively easy handling. Crucially, semiconductor QDs provide the possibility to integrate photonic properties with the spin of an individual magnetic impurity [5]. The magnetic spin can be selectively manipulated and used for information storage [6, 7]. However, the use of semiconductor QDs in a realistic working device requires a reliable control of the excitation process as well as an understanding of the emission channels.

An important, but nevertheless, little investigated recombination channel is related to the dark exciton states i.e. states with total angular momentum equal to 2 [8]. Random transitions between dark and bright excitonic states lead to exciton decoherence [9] and a significant modification of the recombination dynamics which can result in the delayed emission of photons [10, 11]. Despite their importance, dark exciton states are difficult to probe. The radiative recombination of dark excitons is forbidden so that they usually cannot be studied directly using spectroscopic techniques. Their properties can be accessed indirectly by a detailed analysis of the dynamics in time-resolved profiles of the bright exciton photoluminescence $10-12$. The other possibility is to measure the weak optical transitions under conditions in which the dark exciton recombination is partially allowed. This has been achieved either by the use of the in-plane magnetic field which mixes the heavy-light hole states [13, 14] or by placing the QD in a micro-pillar which enhances the coupling of the exciton with light [15].

Here we present an investigation of dark exciton optical transitions which are allowed due to the simultaneous spin flip of coupled single magnetic impurity. We analyze the dark exciton wave function and show that the radiative recombination of dark excitons is efficient only when the exchange interaction with the magnetic ion is accom- panied by mixing of the heavy-light hole states, related to an in-plane anisotropy of the QD. To demonstrate the interplay of both mechanisms, high magnetic field spectroscopy has been employed. We determine all relevant parameters such as the dark exciton oscillator strength, the in-plane anisotropy, and the exchange interaction. Additionally, we show that the dark exciton recombination can be used as an efficient channel for controlling the orientation of the spin of the magnetic ion.

The sample, which was grown using molecular beam epitaxy, contains a single layer of self-assembled CdTe QDs with a low concentration of $\mathrm{Mn}^{2+}$ ions, embedded in a ZnTe matrix. The $\mathrm{Mn}^{2+}$ concentration was adjusted to obtain a significant number of QDs containing exactly one $\mathrm{Mn}^{2+}$ ion [16]. For the measurements, the sample was placed in a micro-photoluminescence ( $\mu$-PL) setup and kept at the temperature of $4.2 \mathrm{~K}$. The resistive magnet produced magnetic field up to $28 \mathrm{~T}$. The field was applied in the Faraday configuration, parallel to the the growth axis of the sample. The PL of the QDs was excited either above the gap of the ZnTe barrier (at $532 \mathrm{~nm}$ ) or using a tunable dye laser in the range $570-610 \mathrm{~nm}$. Both the exciting and the collected light were transmitted though a monomode fiber coupled directly to the microscope objective. The use of the monomode fiber combined with polarization optics outside the cryostat permits the control of the circular polarization of both the exciting and detected light.

The diameter of both the excitation and detection spot was less than $2 \mu \mathrm{m}$, which allows us to select spectra of different single QDs containing a single $\mathrm{Mn}^{2+}$ ion. Representative results for two selected QDs with different inplane anisotropy are shown in the insets of Fig. 1. Characteristic PL spectra contain a neutral exciton $(X)$ lines split into sextuplets due to the $X$-Mn exchange interaction [5, 17]. The total spin of the $\mathrm{Mn}^{2+}$ ion is $5 / 2$, and it has six possible projections onto the direction of highly anisotropic excitonic spin. Thus, each of the six possible spin states of the ion is related to a specific component of 


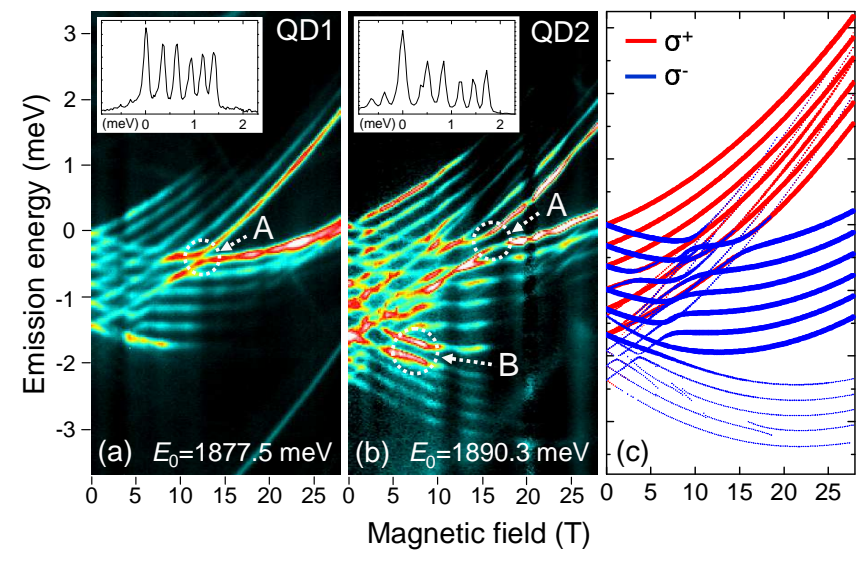

FIG. 1. (a-b) Color-scale plots of the PL spectra of two single Mn-doped QDs as a function of magnetic field. Two dots (QD1 and QD2) differ by in-plane anisotropy. The vertical scale of each panel is shifted by $E_{0}$. Insets: PL spectra at $B=$ 0. (c) Simulation of the optical transitions in the QD2 with the model described in text. The line thickness corresponds to the oscillator strength of the transitions.

the excitonic sextuplet for a given circular polarization. As in our previous work [7], we have selected dots containing a single $\mathrm{Mn}^{2+}$ ion which have a nonmagnetic QD as a close neighbor. The resonant excitation of this dot is followed by a spin conserving $X$ transfer to the Mndoped QD [18]. This permits the efficient and selective excitation of the luminescent dot as well as the selection of the spin polarization of excitons injected into this dot.

Figure 1(a-b) shows the evolution of neutral exciton PL spectra with magnetic field, measured without polarization resolution for the two selected QDs. To elucidate the most characteristic spectral features of this evolution we initially focus on Fig. 1(a) showing the QD with the smaller in-plane anisotropy. In the magnetic field, the exciton sextuplet splits into two distinct Zeeman branches corresponding to $\sigma^{+}$and $\sigma^{-}$circular polarizations for the upper and lower branch respectively. The lowest component of each branch corresponds to the $\mathrm{Mn}^{2+}$ spin antiparallel to the exciton spin [5]. The resonant excitation with $\sigma^{-}$polarization prevents the $\mathrm{Mn}^{2+}$ spin from thermalizing in the magnetic field [7] due to the interaction of the $\mathrm{Mn}^{2+}$ ion with spin-polarized carriers injected into the dot. Therefore all six lines are visible in both branches up to magnetic fields $\sim 15 \mathrm{~T}$.

At a magnetic field of around $12 \mathrm{~T}$, an anticrossing of the outermost lines of both branches is clearly visible ( "A" in Fig1(a)). These two lines correspond to the same $-5 / 2$ state of the $\mathrm{Mn}^{2+}$ spin, but two opposite spin states of $X$. At this field, the excitonic Zeeman splitting exactly compensates the $X-\mathrm{Mn}$ exchange interaction. The splitting (anticrossing) of these two lines is then simply due to the in-plane anisotropy of the $\mathrm{QD}$, acting via the anisotropic component of the electron-hole $(e-h)$ ex- change interaction, as observed for excitonic lines in the absence of magnetic field for nonmagnetic QD [19]. As we have checked, the two split lines show linear polarization (presumably along the symmetry axes of the anisotropic dot), in contrast to the remaining sextuplet lines which are polarized circularly. The anisotropic exchange splitting, determined from the anticrossing, is equal to $60 \mu \mathrm{eV}$ for QD1 in Fig 1(a), and $230 \mu \mathrm{eV}$ for QD2 in Fig 1(b).

Our optical method to align the $\mathrm{Mn}^{2+}$ spin against the action of the external magnetic field [7] becomes less efficient at magnetic fields above $\sim 15 \mathrm{~T}$. This is due to the accelerated spin-lattice relaxation of the $\mathrm{Mn}^{2+}$ spin at high magnetic fields [20]. As a result, the $\mathrm{Mn}^{2+}$ spin orientation thermalizes and the excitonic lines related to the less populated spin states vanish.

Strikingly, the QD with the larger anisotropic exchange splitting value (Fig 1(b)) has an additional, albeit weaker, lower branch consisting of only five lines. To understand the origin of this branch one should notice that optical transitions are possible in two situations: (a) The projection of total angular momentum of the exciton on the quantization axis is equal to \pm 1 (bright exciton). In this case the transition is dipole allowed and the $\mathrm{Mn}^{2+}$ spin projection is conserved during the $X$ recombination. Branches with six lines are related to this kind of recombination. (b) The projection of total angular momentum of the exciton is equal to \pm 2 (dark exciton, $X_{d}$ ). Then, in the first approximation the optical transition is dipole forbidden. However, the valence band mixing and the exchange interaction with the $\mathrm{Mn}^{2+}$ ion result in a mixing of the electron and hole spin states. As a result, the $X_{d}$ states have an admixture of the $X$ states with the $\mathrm{Mn}^{2+}$ spin projection different by 1 . Thus, the $X_{d}$ recombination is possible when accompanied by the simultaneous spin-flip of the $\mathrm{Mn}^{2+}$ ion. As there are only 5 possible transitions between the $6 \mathrm{Mn}^{2+}$ spin states, the PL lines related to $X_{d}$ present a fivefold spitting. The upper energy branch of $X_{d}$ is not clearly visible in our experiment because it overlaps with much stronger $X$ transitions.

The quantum states of the exciton and $\mathrm{Mn}^{2+}$ spin can be described in the basis given by three quantum numbers: $\left|S_{z}, \sigma_{z}, j_{z}\right\rangle$ indicating the $\mathrm{Mn}^{2+}$, electron and hole angular momentum projections onto the quantization axis parallel to the magnetic field. Using the available information for the $\mathrm{g}$-factors of the carriers and the $\mathrm{Mn}^{2+}$ ion 21], we attribute the $X_{d}$ low energy branch to the recombination of $\left|S_{z},+1 / 2,+3 / 2\right\rangle$ states. Therefore, to satisfy the selection rules for $X_{d}$ dipolar recombination the projection of the $\mathrm{Mn}^{2+}$ spin must be increased by 1 . This implies that each possible $X_{d}$ recombination is related to a spin-flip of the $\mathrm{Mn}^{2+}$ ion towards the state polarized opposite to the thermalized state. There are three different admixtures of the $X_{d}$ states which make this process possible (see Fig. 2(a)). The first one is the state with the opposite spin projection of the electron $\left(\left|S_{z}+1,-1 / 2,+3 / 2\right\rangle\right)$. It is mixed with the $X_{d}$ state 

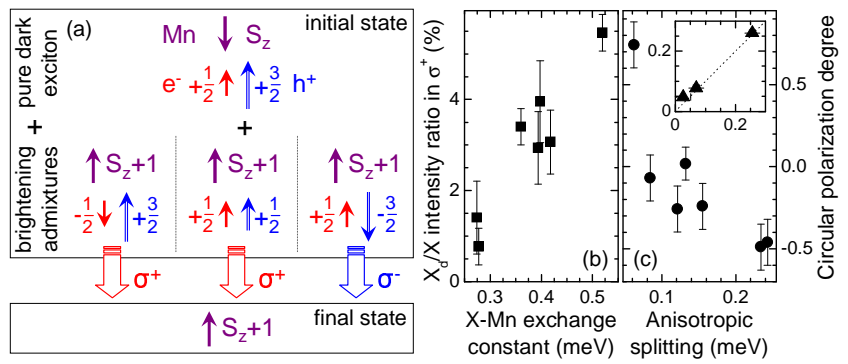

FIG. 2. (a) Schematic diagram of $X$ admixtures in the $X_{d}$ states and possible channels of optical transitions related to the $X_{d}$ recombination. (b) $X_{d} / X$ intensity ratio in $\sigma^{+}$polarization vs. $X-\mathrm{Mn}$ exchange constant. (c) Degree of circular polarization of $X_{d}$ spectrum vs. anisotropic exchange splitting of the QD. A value of -1 denotes pure $\sigma^{-}$polarization, while a value of +1 refers to pure $\sigma^{+}$polarization. Inset: The width of splitting between the $|+1.5,+1 / 2,+3 / 2\rangle$ and $|+2.5,+1 / 2,-3 / 2\rangle$ lines ("B" in Fig. 11(b)) calculated from the model vs. splitting between these lines determined directly from the PL for those QDs for which it was possible.

due to the $e-\mathrm{Mn}$ exchange interaction. The second admixture, caused by the $h$-Mn exchange interaction, consists of a light hole with a spin projection different by 1 from the heavy hole of the $X_{d}\left(\left|S_{z}+1,+1 / 2,+1 / 2\right\rangle\right)$. The last admixed state, $\left|S_{z}+1,+1 / 2,-3 / 2\right\rangle$, consists of a heavy hole with a spin projection different by 3 from the spin projection of the original state. This admixture is induced by two interactions acting together: the heavy-light hole mixing, which mixes hole states with spin projection different by 2 , and by the $h$-Mn exchange interaction. A direct experimental evidence for the presence of valence band mixing is provided by the anticrossing of the highest energy line of $X_{d}$ and the lowest energy line of $X$ at a magnetic field $\sim 7 \mathrm{~T}$ (labeled "B" in Fig 1(b)). These lines correspond to $|+1.5,+1 / 2,+3 / 2\rangle$ and $|+2.5,+1 / 2,-3 / 2\rangle$ states, respectively.

The radiative recombination related to the first two of these admixtures results in the emission of a photon with $\sigma^{+}$polarization. The amplitudes of these admixtures to the $X_{d}$ state depend only on the $e-\mathrm{Mn}$ and $h$ $\mathrm{Mn}$ exchange constants, i.e. on the overlap of the $\mathrm{Mn}^{2+}$ ion and the carrier wave functions. In contrast, the recombination related to the third admixture produces a $\sigma^{-}$polarized photon. The amplitude of this admixture depends not only on the $X$-Mn exchange interaction, but also on the valence band mixing, which, similarly to the anisotropic exchange splitting, results from the in-plane anisotropy of the QD [14, 22 24]. The role of this anisotropy in determining the polarization of the $X_{d}$ lines is clearly visible in our experiment. Fig. 3 shows spectra of the two QDs shown in Fig 1 for similar excitation conditions and magnetic field near the anticrossing of the lines corresponding to $|-2.5,-1 / 2,+3 / 2\rangle$ and $|-2.5,+1 / 2,-3 / 2\rangle$ states ("A" in Fig $1(\mathrm{a}-\mathrm{b})$ ). The $\sigma^{-}$

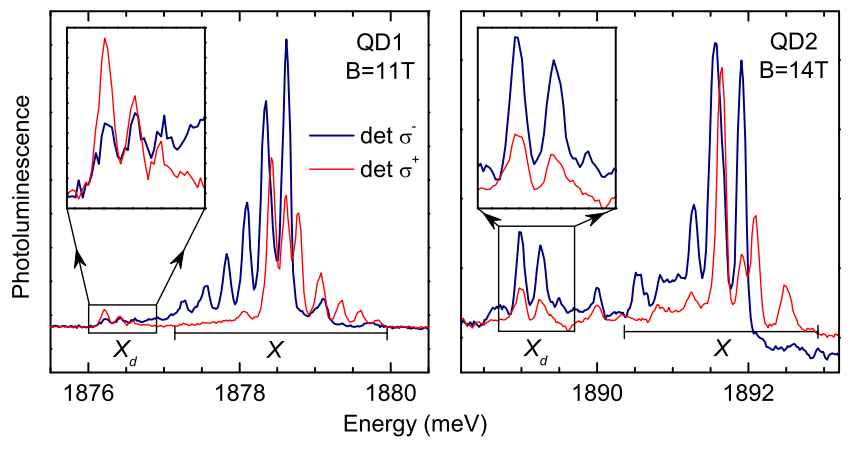

FIG. 3. Spectra of the two QDs shown in Fig. 1 taken under excitation with $\sigma^{-}$polarized light with polarization resolved $\left(\sigma^{+}\right.$or $\left.\sigma^{-}\right)$detection for magnetic fields near the anticrossing of lines corresponding to $|-2.5,-1 / 2,+3 / 2\rangle$ and $|-2.5,+1 / 2,-3 / 2\rangle$ states ("A" in Fig 1 (a-b)).

polarized lines are much more pronounced with respect to the $\sigma^{+}$polarized ones for a highly anisotropic QD2.

As a quantitative measure of the $X_{d}$ oscillator strength for both circular polarizations we use the $X_{d} / X$ intensity ratio. Since this ratio depends on the excitation power 25], one should use identical excitation conditions to be able to compare its value for different QDs. Experimentally, this is achieved by choosing the same $X X / X$ intensity ratio equal in our case to $1 / 3$. The $X X$ intensity increases roughly quadratically with the excitation power, while the $X$ intensity follows linear power dependence $(26,27])$. Thus, this ratio gives a measure of the excitation efficiency.

A strong increase of the $X_{d} / X$ intensity ratio in $\sigma^{+}$ polarization with increasing $X-\mathrm{Mn}$ exchange constant is clearly visible in Fig. 2(b). It confirms the origin of the first two recombination channels described above. However, the $X_{d}$ oscillator strength for $\sigma^{-}$polarization depends both on the $X-\mathrm{Mn}$ exchange interaction and the QD anisotropy. To elucidate the role of the anisotropy, we can use the circular polarization of the $X_{d}$. Fig. 2(c) shows that the polarization of the $X_{d}$ evolves towards $\sigma^{-}$ as the anisotropic exchange splitting increases. Therefore, we conclude that a high in-plane anisotropy of the QD leads to a strong mixing of light and heavy holes, which combined with the presence of the $\mathrm{Mn}^{2+}$ ion is the reason for the strong $\sigma^{-}$polarization and high intensity of the $X_{d}$ spectrum. It is important to note that for nonmagnetic QDs showing similar anisotropy [14] the optical transitions of $X_{d}$ are not observed, except in the presence of a strong in-plane magnetic field.

As each $X_{d}$ recombination in the lower energy branch involves an increase of the $\mathrm{Mn}^{2+}$ spin projection by 1 , dark exciton recombination can play the role of an effective $\mathrm{Mn}^{2+}$ spin orientation mechanism. In our experiment the $X_{d} / X$ intensity ratio for a highly anisotropic $\mathrm{QD}$ and $\sigma^{-}$polarized excitation was as high as $10 \%$. This 
is comparable to the probability of a spin-flip of the $\mathrm{Mn}^{2+}$ ion per one recombination of the $X$ in the QD which was estimated to be $\sim 10 \%$ in Ref. [7]. While this orientation mechanism should be present for both circular polarizations of excitation, only the $\sigma^{-}$polarization which populates the low energy branch of $X_{d}$ is fully seen. The $\sigma^{+}$ polarized excitation decreases the $\mathrm{Mn}^{2+}$ spin projection [7]. Under such conditions the high energy branch of $X_{d}$ should be populated and the low energy branch should be virtually invisible. The latter is, indeed, confirmed in our experiment. However, it is not possible to observe directly the high energy $X_{d}$ branch, since it occurs in the same energy region as the much stronger $X$ lines.

A quantitative description of key features of the data in Fig. 1 (a-b) is provided by a simple model with the initial state of the QD after excitation event given by the following Hamiltonian ([13, $14,[28,30]$ ),

$$
\begin{array}{r}
\mathcal{H}=g_{M n} \mu_{B} \vec{B} \cdot \vec{S}+g_{e} \mu_{B} \vec{B} \cdot \vec{\sigma}+g_{h} \mu_{B} \vec{B} \cdot \vec{j}-I_{e} \vec{S} \cdot \vec{\sigma} \\
-I_{h} \vec{S} \cdot \vec{j}+\sum_{i=x, y, z}\left(a_{i} j_{i} \sigma_{i}+b_{i} j_{i}^{3} \sigma_{i}\right)-\gamma j_{z}^{2}+\beta\left(j_{x}^{2}-j_{y}^{2}\right)
\end{array}
$$

where $S, \sigma$ and $j$ are the $\mathrm{Mn}^{2+}$, electron and hole spin operators, respectively, the first three terms represent the Zeeman energy of the $\mathrm{Mn}^{2+}$ ion, the electron and the hole, $I_{e}$ and $I_{h}$ are the $e-\mathrm{Mn}$ and $h$-Mn exchange interaction constants, $a_{i}$ and $b_{i}$ are $e-h$ spin-spin coupling constants, $2 \gamma$ is the heavy-light hole splitting and $\beta$ represents the strength of the heavy-light hole mixing. The first term is also the Hamiltonian of the final state of the system after the exciton recombination. We also introduced an additional, phenomenological term related to the excitonic diamagnetic shift to facilitate a comparison of the model and experimental data.

The energies of the optical transitions versus magnetic field, calculated using this Hamiltonian for the two circular polarizations, are plotted in Fig. 1(c). The calculations clearly reproduce the key features of the experimental data in Fig. 1(a-b), such as for example the $X$-Mn exchange splitting and the anisotropic exchange splitting. All parameters in the Hamiltonian (except for $\gamma$ assumed to be $15 \mathrm{meV}$ [24]) can be extracted by fitting to the experimental data. In particular, the heavy-light hole mixing can be estimated using the degree of circular polarization of $X_{d}$ lines. Such an approach permits an estimation of the $\beta$ parameter even for those QDs, for which the anticrossing between the $|+1.5,+1 / 2,+3 / 2\rangle$ and $|+2.5,+1 / 2,-3 / 2\rangle$ lines ("B" in Fig. 1 (b)) is not clearly visible. As shown in the inset of Fig. 2(c) the obtained value of this anticrossing remains in very good agreement with the value estimated directly from PL data. This confirms the proposed mechanism of $X_{d}$ brightening.

To conclude, we have used a QD with a single magnetic impurity $\left(\mathrm{Mn}^{2+}\right)$ to investigate the dark exciton transitions. The $X$-Mn exchange interaction, when com- bined with a mixing of the heavy-light hole states induced by the QD in-plane anisotropy, allows dark exciton recombination accompanied by a simultaneous $\mathrm{Mn}^{2+}$ spin flip. High magnetic fields have been used to spectrally separate the $\mathrm{PL}$ lines related to $X$ and $X_{d}$ transitions and to extract the important QD parameters (e.g. the anisotropic exchange splitting). The excitation of the Mn-doped QD via a closely lying second QD permits the precise control of the spin of the carriers injected to the QD and therefore a control over the $\mathrm{Mn}^{2+}$ spin orientation. The simple Hamiltonian, which describes this system, reproduces correctly all the key features of the PL spectra of the QD in high magnetic field.

This work was supported by the Polish Ministry of Science and Higher Education as research grants in years 2006-2011, by the 6th Research Framework Programme of EU (contract MTKD-CT-2005-029671), by the CNRS PICS-4340 Programme and by the Foundation for Polish Science. Two of us (P.P. and P.K.) are supported by the EU under FP7, contracts no. 221249 'SESAM' and no. 221515 'MOCNA' respectively. We thank Anna Trojnar and Marek Korkusiński for fruitful discussions.

* Mateusz.Goryca@fuw.edu.pl

[1] P. Michler et al., Science, 290, 2282 (2000).

[2] P. Michler et al., Nature, 406, 968 (2000).

[3] C. Santori et al., Phys. Rev. Lett., 86, 1502 (2001).

[4] V. Zwiller et al., Appl. Phys. Lett., 78, 2476 (2001).

[5] L. Besombes et al., Phys. Rev. Lett., 93, 207403 (2004).

[6] C. Le Gall et al., Phys. Rev. Lett., 102, 127402 (2009).

[7] M. Goryca et al., Phys. Rev. Lett., 103, 087401 (2009).

[8] J. D. Cuthbert and D. G. Thomas, Phys. Rev., 154, 763 (1967).

[9] P. Palinginis et al., Phys. Rev. B. 70, 073302 (2004)].

[10] S. A. Crooker et al., Appl. Phys. Lett., 82, 2793 (2003).

[11] O. Labeau, P. Tamarat, and B. Lounis, Phys. Rev. Lett., 90, 257404 (2003).

[12] G. Bacher et al., Phys. Rev. Lett., 83, 4417 (1999).

[13] M. Bayer et al., Phys. Rev. B, 65, 195315 (2002).

[14] K. Kowalik et al., Phys. Rev. B, 75, 195340 (2007).

[15] M. Winger et al., Phys. Rev. Lett., 101, 226808 (2008).

[16] P. Wojnar et al., Phys. Rev. B, 75, 155301 (2007).

[17] J. Fernández-Rossier, Phys. Rev. B, 73, 045301 (2006).

[18] T. Kazimierczuk et al., Phys. Rev. B, 79, 153301 (2009).

[19] D. Gammon et al., Science, 273, 87 (1996).

[20] T. Strutz, A. M. Witowski, and P. Wyder, Phys. Rev. Lett., 68, 3912 (1992).

21] L. Besombes et al., J. Cryst. Growth, 214, 742 (2000).

[22] M. Bayer et al., Phys. Rev. Lett.. 82, 1748 (1999).

[23] A. V. Koudinov et al., Phys. Rev. B, 70, 241305 (2004).

[24] Y. Léger et al., Phys. Rev. B, 76, 045331 (2007).

[25] L. Besombes et al., Phys. Rev. B, 71, 161307 (2005).

[26] K. Brunner et al., Phys. Rev. Lett., 73, 1138 (1994)

[27] J. Suffczyński et al., Phys. Rev. B, 74, 085319 (2006)].

[28] H. W. van Kesteren et al., Phys. Rev. B, 41, 5283 (1990).

[29] E. L. Ivchenko, A. Y. Kaminski, and U. Rössler, Phys. Rev. B, 54, 5852 (1996)

[30] A. A. Toropov et al., Phys. Rev. B, 63, 035302 (2000). 